\title{
Tracking defect-induced ferromagnetism in GaN:Gd
}

\author{
Martin Roever, Joerg Malindretos, ${ }^{*}$ Amilcar Bedoya-Pinto, and Angela Rizzi \\ IV. Physikalisches Institut, Georg-August-Universtät Göttingen, Göttingen, Germany \\ Christian Rauch and Filip Tuomisto \\ Department of Applied Physics, Aalto University, Aalto, Finland \\ (Received 22 March 2011; published 10 August 2011)
}

\begin{abstract}
We report on the magnetic properties of $\mathrm{GaN}: \mathrm{Gd}$ layers grown by molecular beam epitaxy. A poor reproducibility with respect to the magnetic properties is found in these samples. Our results show strong indications that defects with a concentration of the order of $10^{19} \mathrm{~cm}^{-3}$ might play an important role for the magnetic properties. Positron annihilation spectroscopy does not support a direct connection between the ferromagnetism and the Ga vacancy in GaN:Gd. Oxygen codoping of GaN:Gd promotes ferromagnetism at room temperature and points to a role of oxygen for mediating ferromagnetic interactions in Gd-doped $\mathrm{GaN}$.
\end{abstract}

DOI: 10.1103/PhysRevB.84.081201

PACS number(s): 71.55.Eq, 76.30.Kg, 75.50.Pp, 78.70.Bj

The doping of nonmagnetic semiconductors by magnetic metal ions ( $3 d$ or $4 f$ ) has been among the popular topics of the past decade in the field of semiconductor spintronics. The aim is to introduce magnetic impurities that provide local spins in a nonmagnetic host and to have a long-range interaction mechanism between them that can lead to ferromagnetism at or above room temperature. Interestingly, also localized defect states can provide extra magnetic moments which might interact either with the magnetic ions or even just among themeselves. ${ }^{1-5}$ In these models the distances between the magnetic moments for a nonvanishing exchange interaction are usually below $\sim 10 \AA$. In this case the percolation threshold that leads to the onset of a macroscopic ferromagnetic state requires concentrations of localized moments of $\sim 1 \%$. ${ }^{6}$ Such high concentrations can usually not be obtained in thermal equilibrium and therefore the experiments require a high level of control and the results must be critically analyzed. Theory has promoted a rush for room-temperature ferromagnetism in the field of dilute magnetic semiconductors. Important aspects and results have been recently reconsidered and critically reviewed by Zunger et al. ${ }^{7}$

The first report on ferromagnetic $\mathrm{GaN}: \mathrm{Gd}$ was on a molecular beam epitaxy (MBE)-grown layer with $\sim 6 \%$ of Gd. ${ }^{8}$ Exceptionally high effective magnetic moments of up to $4000 \mu_{B}$ per $\mathrm{Gd}$ atom were found in highly dilute GaN:Gd layers. ${ }^{9,10}$ Later it was shown that these moments can even be increased to $10^{5} \mu_{B}$ in Gd ion-implanted GaN layers. ${ }^{11}$ Nearly all recent reports about Gd-doped GaN show a strong ferromagnetic signal at room temperature. ${ }^{8-10,12-14}$ Only few exceptions show partly ferromagnetic samples or no ferromagnetism. ${ }^{15,16}$

The origin of the ferromagnetic order in $\mathrm{GaN}: \mathrm{Gd}$ is still unknown. It was shown that cation vacancies can provide local moments which exchange directly by their overlapping wave functions and provide a long-ranged coupling mechanism. ${ }^{1,2}$

Published by the American Physical Society under the terms of the Creative Commons Attribution 3.0 License. Further distribution of this work must maintain attribution to the author(s) and the published article's title, journal citation, and DOI.
According to Neugebauer et al., the cation vacancy in $\mathrm{GaN}$ is a rather unlikely defect if the Fermi energy is not close to the conduction-band minimum. ${ }^{17}$ Furthermore, nitrogen or oxygen interstitials in $\mathrm{GaN}$ :Gd can also provide local moments with a long-ranged coupling and their formation is much more likely than the cation vacancy. ${ }^{4}$

In this Rapid Communication we show experimental results about the reproducibility and long-term stability of the ferromagnetic phase in Gd-doped GaN layers. The theory of the Ga vacancy model is probed experimentally by positron annihilation spectroscopy (PAS). Oxygen codoping of GaN:Gd was carried out to introduce oxygen impurities on purpose.

All GaN:Gd samples were grown by plasma-assisted molecular beam epitaxy on metalorganic chemical vapor deposition (MOCVD) $\mathrm{GaN} / \mathrm{Al}_{2} \mathrm{O}_{3}(0001)$ templates. If not stated otherwise, the MBE process was carried out with our optimized parameters for the growth of unintentionally doped GaN layers, namely, $760{ }^{\circ} \mathrm{C}$ substrate temperature and slightly metal-rich conditions. Gd $(4 N)$ was supplied during growth by a high-temperature effusion cell. Oxygen codoping was carried out applying oxygen gas $(4.8 N)$ with a flux ranging from 0.1 to $0.5 \mathrm{sccm}$ into the growth chamber. The growth time was kept constant for all epitaxial layers, resulting in a thickness of $\sim 500 \mathrm{~nm}$ for the standard GaN:Gd layers and $200 \mathrm{~nm}$ for the oxygen codoped samples.

A superconducting quantum interference device (SQUID) MPMS 5 from Quantum Design was used to measure the magnetic properties. All data have been corrected for diamagnetic background and trapped field artifacts. ${ }^{18,19}$ The resolution limit is $\sim 3 \times 10^{-7} \mathrm{emu}$, which translates to $\sim 5 \times 10^{18} \mu_{B} / \mathrm{cm}^{3}$ for our sample geometry. Reference measurements on the substrate material were made on a regular basis and no ferromagnetic contributions were found.

The Gd concentration is measured by time of flight secondary ion mass spectroscopy. The resolution limit of Gd isotopes is $\sim 10^{16} \mathrm{~cm}^{-3}$. All lower concentrations are extrapolated by effusion theory. The contamination with transition metals can be estimated to be at least a factor of $10^{3}$ lower than the Gd concentration. ${ }^{20}$

The Doppler broadening of the positron annihilation radiation was measured by two germanium detectors with a 

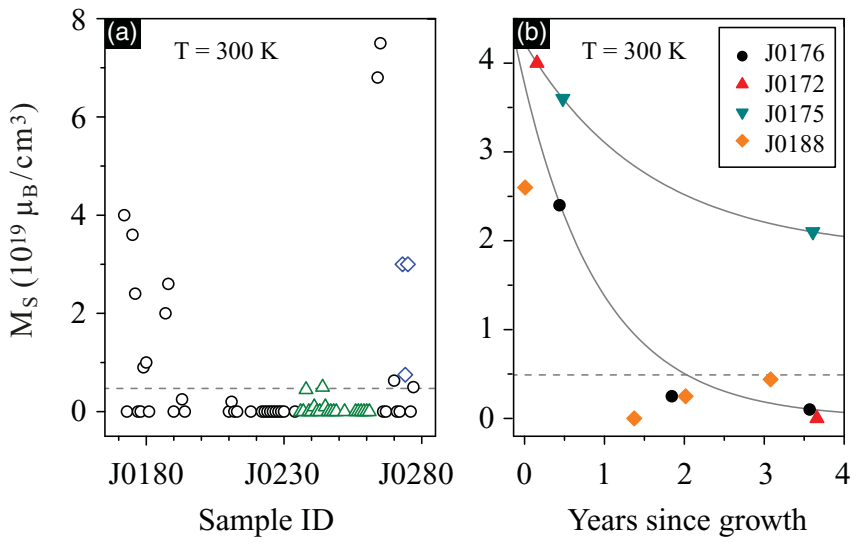

FIG. 1. (Color online) (a) Saturation magnetization at $300 \mathrm{~K}$ against the sample ID. Black circles show Gd-doped GaN samples grown under optimized conditions. Green triangles represent samples grown under varying conditions as explained in the text. Blue diamonds stand for oxygen codoped GaN:Gd. (b) Saturation magnetization against time for selected samples. The dotted line is the resolution limit of the SQUID magnetometer and the exponential curves are shown as a guide to the eye.

resolution of $1.3 \mathrm{keV}$ at $511 \mathrm{keV}$, using a monoenergetic slow positron beam. A spectrum of $n>5 \times 10^{5}$ counts was accumulated for each point. The line shape of the Doppler-broadened annihilation $\gamma$ radiation is analyzed using the conventional line-shape parameters $S\left(\left|E_{\gamma}-511 \mathrm{keV}\right|<\right.$ $0.75 \mathrm{keV})$ and $W\left(2.86 \mathrm{keV}<\left|E_{\gamma}-511 \mathrm{keV}\right|<7.33 \mathrm{keV}\right)$. All points have been normalized to the value of a suitable reference sample where positrons annihilate solely in the delocalized state in the $\mathrm{GaN}$ lattice.

The structural quality of the Gd doped epitaxial layers below a Gd concentration of $10^{20} \mathrm{~cm}^{-3}$, at which GdN clusters are formed, resembles those of unintentionally doped $\mathrm{GaN}$, as deduced from $\mathrm{x}$-ray diffraction analysis as well as transmission electron microscopy (not shown). An x-ray absorption near edge structure (XANES) analysis revealed that the Gd is incorporated substitutional on Ga sites in a 3+ valence state and no significant bond length variations of the $\mathrm{Ga}-\mathrm{N}$ bond was found. ${ }^{21}$

In Fig. 1(a) the saturation magnetization $M_{\mathrm{s}}$ at $300 \mathrm{~K}$ measured for many $\mathrm{GaN}$ :Gd epitaxial layers is plotted against the sample ID, i.e., in chronological order. In all samples the Gd concentration is in the range of $10^{14}-10^{19} \mathrm{~cm}^{-3}$. The series around sample number $\mathrm{J} 0180$ clearly shows sizable $M_{\mathrm{S}}$ values. ${ }^{12}$ On the other hand, several samples that have been grown later with the same nominal growth parameters (black circles) show a vanishing saturation magnetization at room temperature, with the exception of J0264 and J0265. The green triangles correspond to samples grown in a wide range of III-V ratios, slightly different substrate temperatures, and within the same range of $\mathrm{Gd}$ concentrations, but no influence of these parameters on the magnetic properties was found. Oxygen codoping of three samples (blue diamonds) was found to induce a ferromagnetic order at room temperature with $M_{\mathrm{S}}$ values comparable to that of the first series. The saturation magnetization value increases with the oxygen supply during growth. It should be noted that GaN:Gd samples without oxygen codoping grown directly before and after the

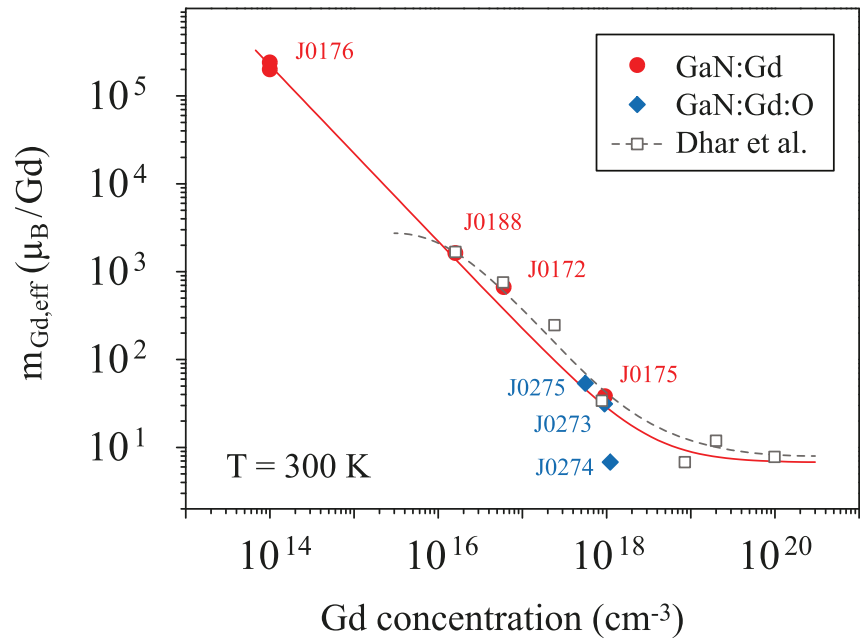

FIG. 2. (Color online) Effective saturation magnetization of ferromagnetic GaN:Gd samples against Gd concentration. Red dots and blue diamonds are samples of this work; for comparison, the data from Dhar et al. is shown as open squares (Ref. 10). The red line represents a fit as discussed in the text.

codoped samples exhibit no ferromagnetism. Selected samples have been repeatedly characterized by SQUID. The saturation magnetization is observed to decrease with time [Fig. 1(b)].

A first general result from these experiments is a very poor reproducibility of the magnetic behavior. Thus, uncontrolled parameters seem to be responsible for the ferromagnetic properties in the GaN:Gd epitaxial layers grown by MBE. In particular, the role played by either intrinsic or extrinsic point defects has to be taken into account. It is worth noting that the presumed defects responsible for the ferromagnetic properties are only induced if Gd is supplied and incorporated into the layers during growth, since none of our undoped GaN layers shows ferromagnetism.

Ferromagnetic and nonferromagnetic samples have been characterized by photoluminescence at $2 \mathrm{~K}$ and the main observation is a clear enhancement of the defect related luminescence for the ferromagnetic GaN:Gd layers (not shown), but no clear assignment is possible. The role of defects was also pointed out in experiments with Gd-implanted $\mathrm{GaN}$ and as-grown GaN:Gd epitaxial layers. ${ }^{11,14,22}$

The dependence of the effective saturation magnetization per Gd atom $m_{\mathrm{Gd}, \text { eff }}$ on the Gd concentration $c_{\mathrm{Gd}}$ is depicted in Fig. 2 and compares well with values in the literature. ${ }^{10}$ The observed trend could be described by a constant additional magnetic moment $M_{\text {def }}=2.5 \times 10^{19} \mu_{B} / \mathrm{cm}^{3}$ independent of the Gd concentration, according to the expression $m_{\mathrm{Gd}, \text { eff }}=$ $m_{\mathrm{Gd}}+M_{\mathrm{def}} / c_{\mathrm{Gd}}$. Assuming a defect-driven mechanism of the ferromagnetism, an effective defect concentration of this order would be required. In this scenario colossal magnetic moments could be explained with a high but not unrealistic concentration of magnetically active defects. However, it is unclear why these defects only form in the presence of Gd while their amount would be independent of the Gd concentration.

Electrical-transport properties of GaN:Gd grown on highly resistive $6 \mathrm{H}-\mathrm{SiC}(0001)$ substrates show that even Gd concentrations lower than the background carrier concentration of typically $10^{17} \mathrm{~cm}^{-3}$ increase the resistivity by several orders 


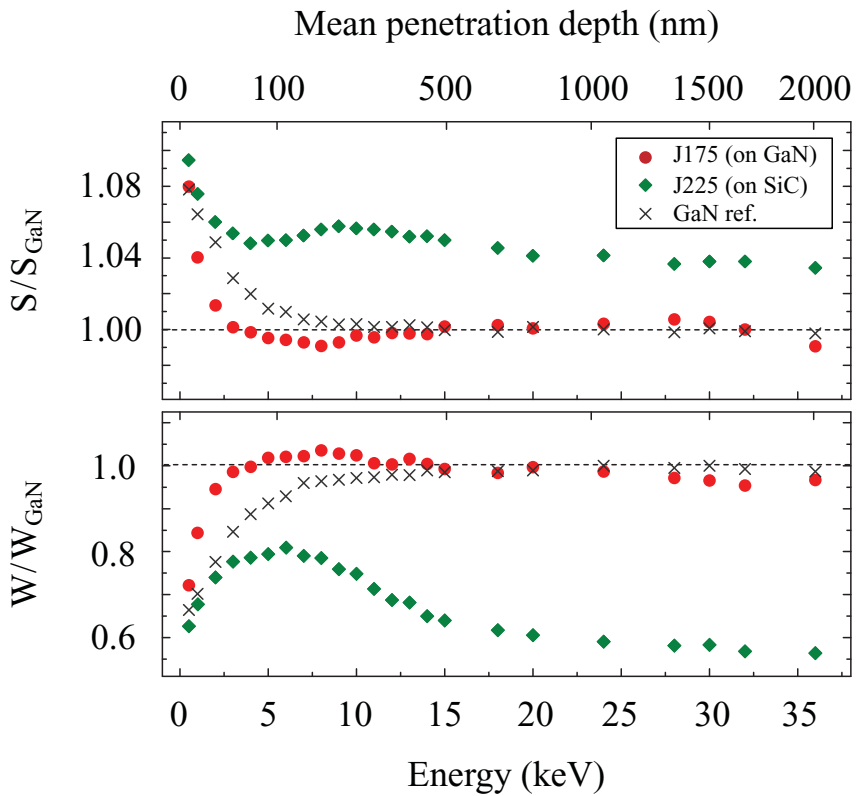

FIG. 3. (Color online) $S$ and $W$ parameters for samples J0175 (magnetic) and J0225 (nonmagnetic) as a function of the positron implantation energy. All spectra are divided by the GaN reference value (see the crossed spectrum for comparison).

of magnitude as compared to unintentionally doped GaN. ${ }^{20}$ The observed variable-range hopping in an impurity band of localized states is another hint that deep defects form in a large number, even for low Gd concentrations.

Secondary ion mass spectroscopy (not shown) reveals oxygen concentrations above $5 \times 10^{18} \mathrm{~cm}^{-3}$ in our GaN:Gd layers (J0175, J0188) and clearly higher values for the oxygen codoped samples (J0273-J0275). The high resistivity of the GaN:Gd layers indicates that the oxygen atoms are not mainly incorporated substitutionally on nitrogen sites, where they would form shallow donors.

With the aim of investigating possible correlations between ferromagnetism in GaN:Gd and the presence of cation vacancies, we performed positron annihilation spectroscopy (PAS). Depth-dependent Doppler broadening spectra have been recorded at room temperature (Fig. 3). From these, the $S$ and $W$ line-shape parameters representative for the GaN:Gd layers are determined and displayed in Fig. 4, together with characteristic values for the GaN lattice and the gallium vacancy. ${ }^{23}$ In order to ensure that the determined parameters at room temperature are representative for the vacancy concentration in the samples and not influenced by positrons detrapping from vacancies to negative ions, ${ }^{24}$ temperature-dependent measurements up to $550 \mathrm{~K}$ have been performed for selected samples (Fig. 4, small triangles). The reference value for the $\mathrm{GaN}$ lattice is determined by measuring a reference sample in which no positron trapping to vacancy defects is observed (Fig. 3).

The identity of dominant positron traps in a certain sample can be evaluated based on its position in the $S W$ plot. The latter is given as the linear combination of the characteristic values of the involved annihilation states, weighted with the fraction of positrons annihilating in these states. The measured $S W$ points for the GaN:Gd layers indicate a broad defect landscape in the

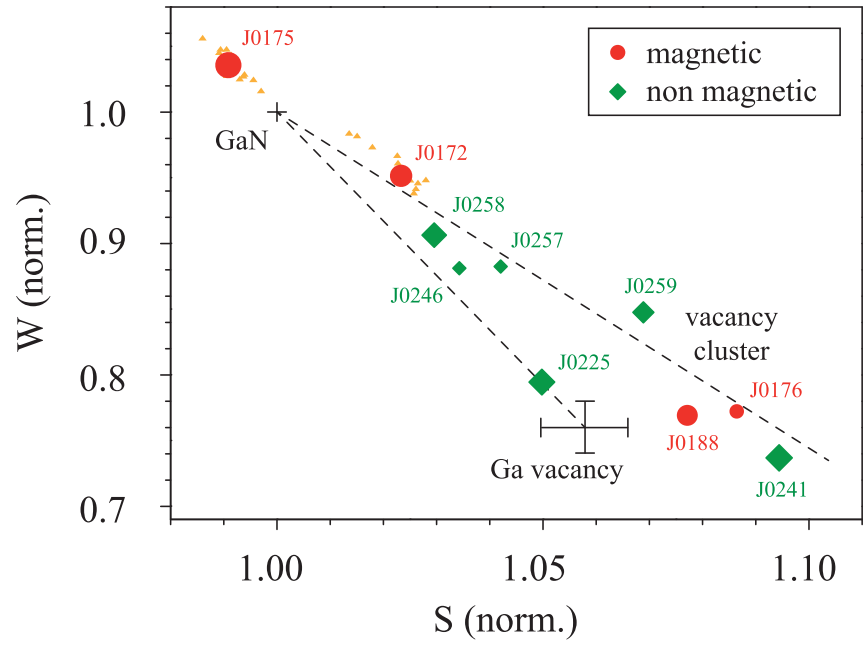

FIG. 4. (Color online) $S W$ plot for the layer-specific line-shape parameters of selected samples at room temperature. Results from temperature-dependent measurements are marked with small triangles. The point size scales with the Gd concentration. Characteristic values for the Ga vacancy (Ref. 23) and vacancy clusters (Ref. 25) are included.

different samples which is dominated by trapping to vacancy clusters. The strong deviation from the Ga vacancy line and high relative $S$ parameters of up to 1.09 are characteristic for vacancy clusters with a considerably larger open volume than the isolated Ga vacancy. The relevant vacancy cluster point is not known exactly, but similar $S$ parameters have been observed before in MBE-grown GaN. ${ }^{25}$ We can estimate the vacancy cluster density to lie in the range between $1 \times$ $10^{16} \mathrm{~cm}^{-3}$ and $1 \times 10^{18} \mathrm{~cm}^{-3}$. Since the accurate $S W$ reference point for these defects is not known, the upper bound could even be lower by about one order of magnitude.

Considering only the ferromagnetic samples in Fig. 4 (red circles), the strongest cluster signal is found for the sample with the lowest Gd concentration $\left(\mathrm{J} 0176, c_{\mathrm{Gd}}=1.5 \times 10^{14} \mathrm{~cm}^{-3}\right)$ and the cluster signal decreases with increasing Gd doping. In the sample with the most stable ferromagnetism with time and highest absolute saturation magnetization (J0175, $c_{\mathrm{Gd}}=9.6 \times 10^{17} \mathrm{~cm}^{-3}$ ), the measured $S W$ point is located close to the $\mathrm{GaN}$ lattice and hence no positron trapping to vacancy defects is observed. At room temperature the positron diffusion length in this sample is considerably reduced compared to the GaN reference (Fig. 3). This hints at the presence of a high concentration of negatively charged ions, ${ }^{24}$ which can trap positrons through the formation of Rydberg states and possess annihilation characteristics close to the $\mathrm{GaN}$ lattice. It should be noted that detrapping from vacancy defects could be explicitly excluded in this sample, due to the temperature-dependent measurements. For the nonmagnetic samples the PAS results do not evidence any clear correlation between the $\mathrm{Gd}$ concentration and the vacancy type or concentration. Sample J0225, whose Gd content of $5.4 \times$ $10^{17} \mathrm{~cm}^{-3}$ is comparable to that of $\mathrm{J} 0175$, lies close to the saturation point for single Ga vacancy-related defects. Nevertheless, this sample never showed any ferromagnetic behavior. So, the defect landscape that has been probed by PAS, namely, single gallium vacancies and larger open 
volume defects, does not directly correlate with the magnetic properties of the GaN:Gd epitaxial layers. We can therefore conclude that the cation vacancy models proposed in literature are not supported by our experiments. ${ }^{1-3}$

Based on formation energy arguments, interstitial nitrogen as well as oxygen in octahedral sites next to $\mathrm{Gd}$ have been proposed to be a more likely source of defect-induced magnetism in $\mathrm{GaN}: \mathrm{Gd}$ than cation vacancies. ${ }^{4}$ Both types of interstitials induce a spin split band of localized states originating from the nonbonding $\mathrm{N}-2 p$ or $\mathrm{O}-2 p$ orbitals, which lies well within the energy gap. Both configurations would be consistent with the observed compensation of the unintentional $n$-type dopants in GaN:Gd and with the electrical transport by hopping. ${ }^{20}$ Due to the large affinity of Gd to $\mathrm{O}$, it is also possible that oxygen donors are attracted into interstitial positions by the $\mathrm{Gd}$, thus further contributing to the compensation and providing extra localized magnetic moments. The efficient gettering of residual donors in $\mathrm{GaN}$ by Gd was also reported for bulk samples grown under high pressure $^{26}$ and was theoretically confirmed by Mitra and Lambrecht for the case of substitutional oxygen. ${ }^{4}$

The nonreproducibility of the ferromagnetic properties in our samples could be explained by an uncontrolled balance between the native donors and the compensating defects. If the concentration of localized magnetic moments were near the percolation threshold, small variations of their amount could switch the macroscopic ferromagnetic state, as discussed in the Appendix of Osorio-Guillen et $a l^{6}$ In this case the favorable effect of codoping with oxygen during growth, as seen for the samples in Fig. 1 (blue diamonds), might be explained by an increase of interstitial oxygen in presence of Gd. Therefore, the model of a ferromagnetic state induced by interstitial oxygen is at least qualitatively consistent with our data. It should be noted that attributing the favorable effect of oxygen codoping to carrier induced ferromagnetism due to $\mathrm{O}_{\mathrm{N}}$ donors seems rather unlikely considering the high resistivity of ferromagnetic $\mathrm{GaN}: \mathrm{Gd} .{ }^{9,20}$

In conclusion, room-temperature ferromagnetism and colossal magnetic moments of dilute GaN:Gd layers grown by $\mathrm{MBE}$ are confirmed. However, the reproducibility and the long-term stability of the magnetic properties are poor. Uncontrolled parameters seem to be responsible for the ferromagnetic properties. It is shown that a concentration of magnetically active defects of $\sim 10^{19} \mathrm{~cm}^{-3}$ might explain the observed magnetic properties. PAS measurements rule out single gallium vacancies as the origin of magnetic coupling in as-grown GaN:Gd and do not show any direct connection of gallium vacancy clusters to room-temperature ferromagnetism in this material either. Oxygen codoping is observed to promote ferromagnetism in GaN:Gd. However, more investigations are necessary to indubitably identify the role of oxygen and the magnetic coupling mechanism in this material.

We thank Andreas Laufer (Justus-Liebig-Universität Gießen) for the SIMS measurements and the Deutsche Forschungs Gesellschaft for funding within the SFB 602. *malindretos@ph4.physik.uni-goettingen.de

${ }^{1}$ P. Dev, Y. Xue, and P. Zhang, Phys. Rev. Lett. 100, 117204 (2008).

${ }^{2}$ Y. Gohda and A. Oshiyama, Phys. Rev. B 78, 161201 (2008).

${ }^{3}$ L. Liu, P. Y. Yu, Z. Ma, and S. S. Mao, Phys. Rev. Lett. 100, 127203 (2008).

${ }^{4}$ C. Mitra and W. R. L. Lambrecht, Phys. Rev. B 80, 081202 (2009).

${ }^{5}$ P. Dev and P. Zhang, Phys. Rev. B 81, 085207 (2010).

${ }^{6}$ J. Osorio-Guillen, S. Lany, S. V. Barabash, and A. Zunger, Phys. Rev. B 75, 184421 (2007).

${ }^{7}$ A. Zunger, S. Lany, and H. Raebiger, Physics 3, 53 (2010).

${ }^{8}$ N. Teraguchi, A. Suzuki, Y. Nanishi, Y.-K. Zhou, M. Hashimoto, and H. Asahi, Solid State Commun. 122, 651 (2002).

${ }^{9}$ S. Dhar, O. Brandt, M. Ramsteiner, V. F. Sapega, and K. H. Ploog, Phys. Rev. Lett. 94, 037205 (2005).

${ }^{10}$ S. Dhar, L. Perez, O. Brandt, A. Trampert, K. H. Ploog, J. Keller, and B. Beschoten, Phys. Rev. B 72, 245203 (2005).

${ }^{11}$ S. Dhar, T. Kammermeier, A. Ney, L. Perez, K. H. Ploog, A. Melnikov, and A. D. Wieck, Appl. Phys. Lett. 89, 062503 (2006). ${ }^{12}$ M. Roever, D.-D. Mai, A. Bedoya-Pinto, J. Malindretos, and A. Rizzi, Phys. Status Solidi C 5, 2352 (2008).

${ }^{13}$ Y. K. Zhou, S. W. Choi, S. Emura, S. Hasegawa, and H. Asahi, Appl. Phys. Lett. 92, 062505 (2008).
${ }^{14}$ R. P. Davies, B. P. Gila, C. R. Abernathy, S. J. Pearton, and C. J. Stanton, Appl. Phys. Lett. 96, 212502 (2010).

${ }^{15}$ J. K. Hite, R. M. Frazier, R. P. Davies, G. T. Thaler, C. R. Abernathy, S. J. Pearton, J. M. Zavada, E. Brown, and U. Hommerich, J. Electron. Mater. 36, 391 (2007).

${ }^{16}$ F.-Y. Lo et al., Appl. Phys. Lett. 90, 262505 (2007).

${ }^{17}$ J. Neugebauer and C. G. Van de Walle, Phys. Rev. B 50, 8067 (1994).

${ }^{18}$ Quantum Design, MPMS Application Note 1014-213, 2002.

${ }^{19}$ Quamtum Design, MPMS Application Note 1014-208, 2001.

${ }^{20}$ A. Bedoya-Pinto, J. Malindretos, M. Roever, D.-D. Mai, and A. Rizzi, Phys. Rev. B 80, 195208 (2009).

${ }^{21}$ G. Martinez-Criado et al., Appl. Phys. Lett. 93, 021916 (2008).

${ }^{22}$ J. Mishra, S. Dhar, and O. Brandt, Solid State Commun. 150, 2370 (2010).

${ }^{23}$ F. Tuomisto, A. Pelli, K. M. Yu, W. Walukiewicz, and W. J. Schaff, Phys. Rev. B 75, 193201 (2007).

${ }^{24}$ K. Saarinen et al., Phys. Rev. Lett. 79, 3030 (1997).

${ }^{25}$ M. Rummukainen, J. Oila, A. Laakso, K. Saarinen, A. J. Ptak, and T. H. Myers, Appl. Phys. Lett. 84, 4887 (2004).

${ }^{26}$ Z. Lipinska, M. Pawlowski, H. Zolnierowicz, A. Wysmolek, M. Palczewska, M. Kaminska, A. Twardowski, M. Bockowski, and I. Grzegory, Acta Phys. Pol. A 110, 243 (2006). 